
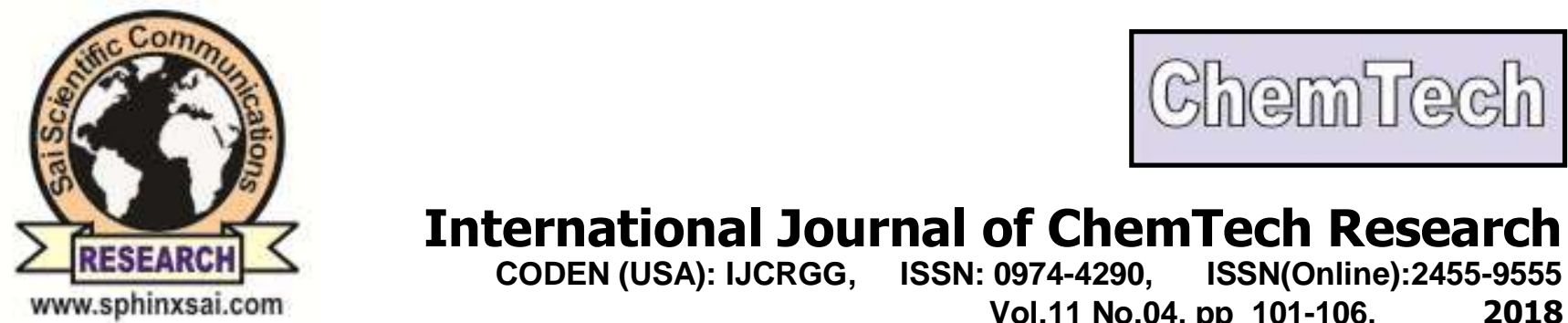

International Journal of ChemTech Research CODEN (USA): IJCRGG, ISSN: 0974-4290, ISSN(Online):2455-9555

Vol.11 No.04, pp 101-106,

2018

\title{
Arduino Based Intelligent Parking Assistance System
}

\author{
S.Baskaran ${ }^{1 *}$, D.Lakshmi ${ }^{2}$, S.Benisha ${ }^{3}$, R.Zahira ${ }^{4}$ \\ 'Department of EEE, S A Engineering College, India \\ ${ }^{2,3}$ Department of EEE, Sree Sastha Institute of Engineering and Tech, India \\ ${ }^{4}$ Department of EEE, Tagore engineering college, India
}

\begin{abstract}
Parking has become an major problem in metro cities like Chennai, Mumbai, and other big cities, especially for the parking spaces for hotels, restaurants, and movie theatres. So the aim of the project is to design an intelligent system that keeps a track of vacant parking spaces and shows the route to those specific parking space locations to avoid wastage of time and fuel to find an empty spot in a parking lot. The car will enter the parking entrance. Once car arrives, sensors will be activated to represent arrival of new vehicle. Then the microcontroller checks for availability of parking space. If there is no vacant parking space, then the gate doesn't open and displays no vacant space message on the LCD board. If parking space is available, then the system allots the parking space nearer to the exit. Once the parking space is allotted, led sign boards will show direction to the driver as of where to park. Once the car is parked, the system updates the number of vacant spaces as reduced by one. Similarly, when a car leaves the parking space, the system detects which space is now empty and increases the vacant space by one.
\end{abstract}

Keywords : Arduino Technology, Intelligent Parking, Microcontrollers, Infrared sensor, obstacle sensing.

\section{Introduction}

As the number of vehicle constantly increases and the resources provided by current parking infrastructures are also limited, vehicle parking has become an important issue. Hence we focus on the optimization of better parking systems mostly in metro cities using an Arduino based intelligent parking assistance system which tries to reduce the confusion caused during parking and which eventually saves the time and cost of the fuel to a greater extent. The prototype contains sensors and Arduino microcontroller to keep track on the number of vehicles. Based on the vehicle count the microcontroller takes decisions and updates the vacant spaces on the LCD screen and assists the car driver as where to park which is indicated by the corresponding LED mounted near every parking slot.

When a vehicle approaches the entry gate, the Entry IR sensor-1 send a signal to Arduino1 and the Arduino1 sends a command to operate the servo motor by $90 \mathrm{deg}$, Subject to space availability is greater than or equivalent to vehicle approaches Entry IR sensor $-2^{1}$. This sends a signal to Micro controller and the gate is closed and the available space is decremented. When the space becomes zero, the Micro controller inhibits the

\section{S.Baskaran et al /International Journal of ChemTech Research, 2018,11(04): 101-106.}


entry gate servo motor. After passing the Entry IR sensor-2, the car approaches to Parking IR sensor-5, 6 and 7 it sends a signal to the Arduino2, hence Arduino2 assists the driver the place of parking with the help of Led at the respective parking lot mostly near the exit. When any of the vehicles goes out, first it approaches the Exit IR sensor-1. This sensor sends a signal to Arduino1 and the Arduinol commands the Exit servo motor to rotate by $90^{\circ}$. When the vehicle approach Exit IR sensor -2 the gate is closed and the available space is incremented in the microcontroller.

\section{Arduino and its Architecture}

The one of the easy and open source of using software and hardware is by Arduino. By using Arduino boards we are able to read inputs - light on a sensor, a finger on a button, or a Twitter message - and convert to output - activating a motor, turning on an LED, publishing something online ${ }^{2}$. It's become very easy way to communicate with board by sending a set of instructions to the microcontroller on the board. It uses the hardware program in Arduino (based on Wiring), and the Arduino Software (IDE), based on Processing. Arduino contain 14 digital input/output pins (of which 6pins are PWM outputs pins), 6 analog inputs, a power jack, a $16 \mathrm{MHz}$ quartz crystal, an ICSP header and reset button, a USB connection ${ }^{2}$. It has everything that are required for the support microcontroller; It should be connected to the computer using USB cable and power supplied using AC-to-DC adapter or power storage battery to get operated.

There are many types of microcontrollers and their platforms are available for physical computation. MIT's Handyboard, Netmedia's BX-24, Phidgets Parallax Basic Stamp, and many others same functionality ${ }^{3}$. All of these tools uses the messy details of microcontroller programming and wrap it up in an easy way of using those package. Arduino also reduces the process of working with microcontrollers, but it provides advantage for teachers, students, and interested amateurs on other systems.

In Fig.2 pins 1 (TX) and 0 (RX) Used to receive (RX) TTL serial data and transmit (TX) TTL serial data. The pins are connected to the corresponding pin of the ATmega8U2 USB-to-TTL Serial chip ${ }^{4}$. Pins 2 and 3 were used to trigger an interrupt to a low value, a change in value or a rising, or falling edge See the attach Interrupt () function ${ }^{5}$. Pin numbers such as $3,5,6,9,10$, and 11 will provide 8-bit PWM output with analog Write () functions. These pin supports SPI communication using the SPI libraries.

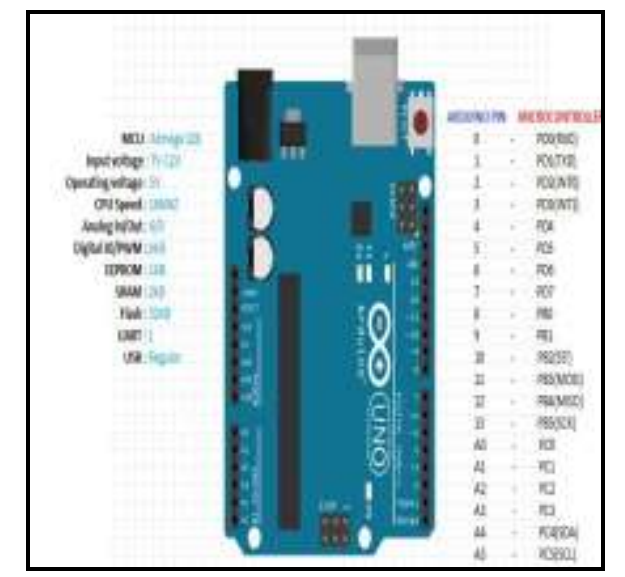

Figure 2: Pin diagram of Arduino board

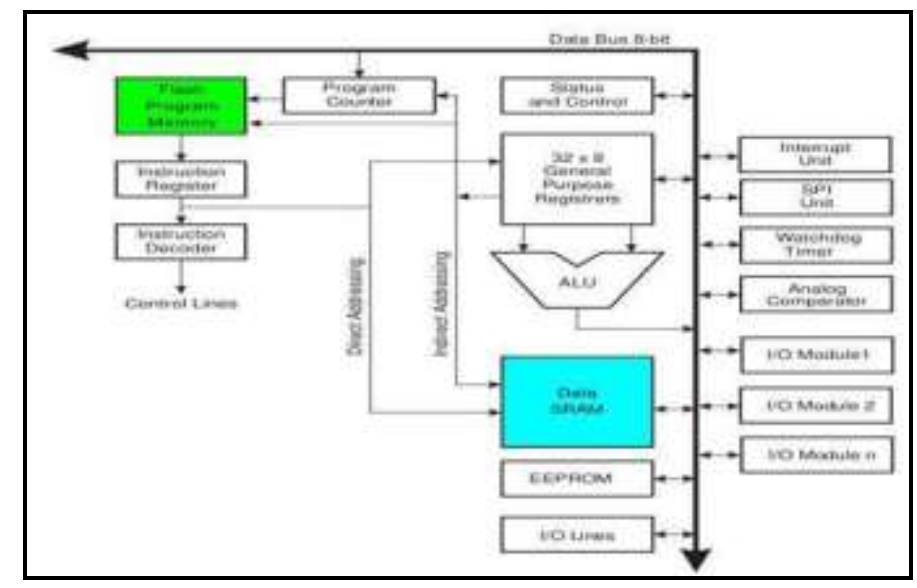

Figure 3: Architecture of Arduino

Harvard architecture was used in the processor of Arduino board where the program code and program data have separate memory. It consists of two memories such as program memory and data memory. Whereas, in data memory data is stored and the code is stored in the flash program memory. The Atmega328 microcontroller has $32 \mathrm{~kb}$ of flash memory, $2 \mathrm{~kb}$ of SRAM $1 \mathrm{~kb}$ of EPROM and operates with a $16 \mathrm{MHz}$ clock speed. The architecture of Arduino is shown in Fig.3. The Advantages of Arduino Technology is It is an open supply hardware feature that permits users to develop own kit. The software is well-suited with all kinds of in operation systems like Windows, Macintosh and Linux, etc. It also comes with the open supply system feature that permits the software system developers to use Arduino code and merge with the prevailing programming language and can be extended and altered. For beginners, it is very easy to use. 


\section{Sensing Circuit}

An infrared sensor is an electronic device that emits in order to sense some aspects of the surroundings. An IR sensor can measure the heat of an object as well as detects the motion. These types of sensors measure only infrared radiation, rather than emitting it that is called as a passive IR sensor. Usually in the infrared spectrum, all the objects radiate some form of thermal radiations. These types of radiations are invisible to our eyes that can be detected by an infrared sensor. The emitter is simply an IR LED (Light Emitting Diode) and the detector is simply an IR photodiode which is sensitive to IR light of the same wavelength as that emitted by the IR LED. When IR light falls on the photodiode, the resistances and these output voltages, change in proportion to the magnitude of the IR light received.

Infrared technology addresses a wide variety of wireless applications. The main areas are sensing and remote controls. In the electromagnetic spectrum, the infrared portion is divided into three regions: near infrared region, mid infrared region and far infrared region. The frequency range of infrared is higher than microwave and lesser than visible light. For optical sensing and optical communication, photo optics technologies are used in the near infrared region as the light is less complex than RF when implemented as a source of signal. Optical wireless communication is done with IR data transmission for short range applications. An infrared sensor emits and/or detects infrared radiation to sense its surroundings. The basic concept of an Infrared Sensor which is used as Obstacle detector is to transmit an infrared signal, this infrared signal bounces from the surface of an object and the signal is received at the infrared receiver.

There are five basic elements used in a typical infrared detection system: an infrared source, a transmission medium, optical component, infrared detectors or receivers and signal processing. Infrared lasers and Infrared LED's of specific wavelength can be used as infrared sources. The three main types of media used for infrared transmission are vacuum, atmosphere and optical fibres. Optical components are used to focus the infrared radiation or to limit the spectral response. Optical lenses made of Quartz, Germanium and Silicon are used to focus the infrared radiation. Infrared receivers can be photodiodes, phototransistors etc. some important specifications of infrared receivers are photosensitivity, detectivity and noise equivalent power. Signal processing is done by amplifiers as the output of infrared detector is very small.

Infrared sensors can be passive or active. Passive infrared sensors are basically Infrared detectors. Passive infrared sensors do not use any infrared source and detects energy emitted by obstacles in the field of view. They are of two types: quantum and thermal. Thermal infrared sensors use infrared energy as the source of heat and are independent of wavelength. Thermocouples, pyroelectric detectors and bolometers are the common types of thermal infrared detectors. Quantum type infrared detectors offer higher detection performance and are faster than thermal type infrared detectors. The photosensitivity of quantum type detectors is wavelength dependent. Quantum type detectors are further classified into two types: intrinsic and extrinsic types. Intrinsic type quantum detectors are photoconductive cells and photovoltaic cells. Active infrared sensors consist of two elements: infrared source and infrared detector. Infrared sources include an LED or infrared laser diode. Infrared detectors include photodiodes or phototransistors. The energy emitted by the infrared source is reflected by an object and falls on the infrared detector. Fig 4 shows the IR sensor circuit.

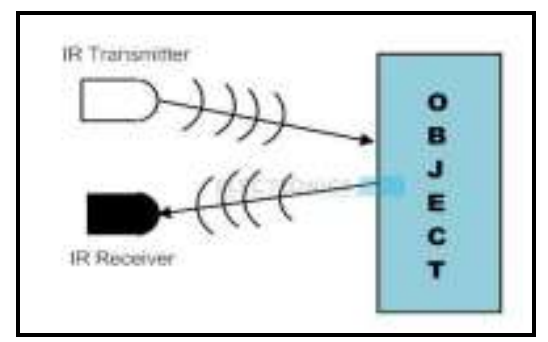

\section{Figure 4: I R Sensor Circuit}

An infrared sensor circuit is one of the basic and popular sensor modules in an electronic device. This sensor is analogous to human's visionary senses, which can be used to detect obstacles and it is one of the common applications in real time. In this paper, the transmitter section includes an IR sensor, which transmits continuous IR rays to be received by an IR receiver module. 
An IR output terminal of the receiver varies depending upon its receiving of IR rays. Since this variation cannot be analyzed as such, therefore this output can be fed to a comparator circuit. Here an operational amplifier (op-amp) of LM 358 is used as comparator circuit. When the IR receiver does not receive a signal, the potential at the inverting input goes higher than that non-inverting input of the comparator IC (LM358). Thus the output of the comparator goes low, but the LED does not glow. When the IR receiver module receives signal to the potential at the inverting input goes low. Thus the output of the comparator (LM 339) goes high and the LED starts glowing. Resistor R1 (100), R2 (10k ) and R3 (330) are used to ensure that minimum $10 \mathrm{~mA}$ current passes through the IR LED Devices like Photodiode and normal LEDs respectively. Resistor VR2 (preset=5k) is used to adjust the output terminals. Resistor VR1 (preset=10k) is used to set the sensitivity.

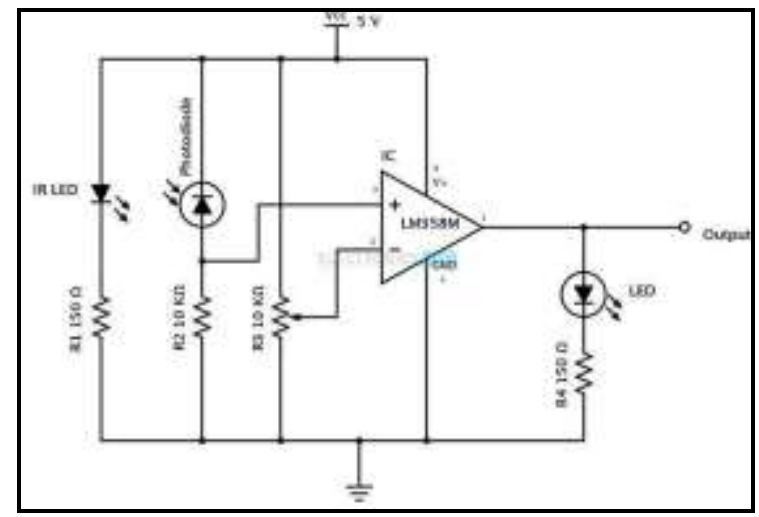

\section{Figure 5: Obstacle sensing circuit}

Fig 5 shows the circuit diagram for obstacle sensing. It consists of an IR LED, a photodiode, a potentiometer, an IC Operational amplifier and an LED.IR LED emits infrared light. The Photodiode detects the infrared light. An IC Op- Amp is used as a voltage comparator. The potentiometer is used to calibrate the output of the sensor according to the requirement. When the light emitted by the IR LED is incident on the photodiode after hitting an object, the resistance of the photodiode falls down from a huge value. One of the input of the op - amp is at threshold value set by the potentiometer. The other input to the op-amp is from the photodiode's series resistor. When the incident radiation is more on the photodiode, the voltage drop across the series resistor will be high. In the IC, both the threshold voltage and the voltage across the series resistor are compared. If the voltage across the resistor series to photodiode is greater than that of the threshold voltage, the output of the IC Op - Amp is high. As the output of the IC is connected to an LED, it lightens up. The threshold voltage can be adjusted by adjusting the potentiometer depending on the environmental conditions.

The positioning of the IR LED and the IR Receiver is an important factor. When the IR LED is held directly in front of the IR receiver, this setup is called Direct Incidence. In this case, almost the entire radiation from the IR LED will fall on the IR receiver. Hence there is a line of sight communication between the infrared transmitter and the receiver. If an object falls in this line, it obstructs the radiation from reaching the receiver either by reflecting the radiation or absorbing the radiation.

\section{Servo Motor Theory}

There are some special types of application of electrical motor where rotation of the motor is required for just a certain angle not continuously for long period of time. For these applications some special types of motor are required with some special arrangement which makes the motor to rotate a certain angle for a given electrical input (signal). For this purpose, servo motor comes into picture. This is normally a simple DC motor which is controlled for specific angular rotation with help of additional servomechanism (a typical closed loop feedback control system). Now day's servo system has huge industrial applications. A servo system mainly consists of three basic components - a controlled device, an output sensor, a feedback system.

This is an automatic closed loop control system. Here instead of controlling a device by applying variable input signal, the device is controlled by a feedback signal generated by comparing output signal and reference input signal. When reference input signal or command signal is applied to the system, it is compared with output reference signal of the system produced by output sensor, and a third signal produced by feedback 
system. This third signal acts as input signal of controlled device. This input signal to the device presents as long as there is a logical difference between reference input signal and output signal of the system. After the device achieves its desired output, there will be no longer logical difference between reference input signal and reference output signal of the system. Then, third signal produced by comparing theses above said signals will not remain enough to operate the device further and to produce further output of the system until the next reference input signal or command signal is applied to the system. Hence the primary task of a servomechanism is to maintain the output of a system at the desired value in the presence of disturbances.

\section{Arduino Coding}

An Arduino is an single microcontroller and a software suite for the programming of it. The hardware consists of a simple open hardware design for the controller with an Atmel AVR processor and on-board I/O support. The software consists of a standard programming language and the boot loader that runs on the board."

\section{Hardware Prototype}

The prototype consists of several components such as the Arduino development board, sensors, and servomotors. The fig 6,7,8, and 9 shows the hardware prototype. The program is flashed to the Arduino which in turns process the program and result in working of the corresponding hardware components. The LED in the parking lot glows according to the parking space available. The simulation results are detection of the number of vehicles and the area of each vehicle.

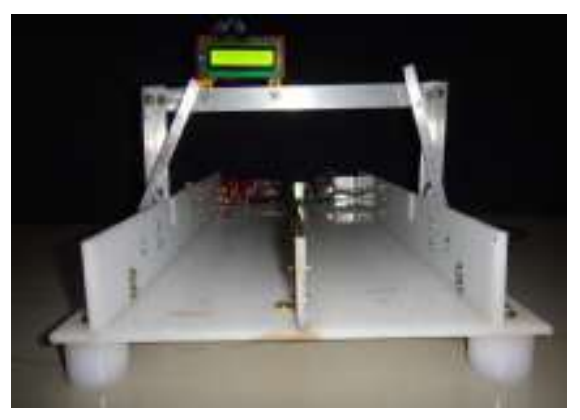

Figure 6: Model of parking system

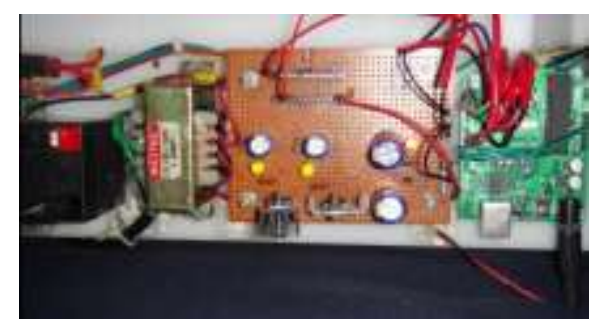

Figure 8: Circuit Board

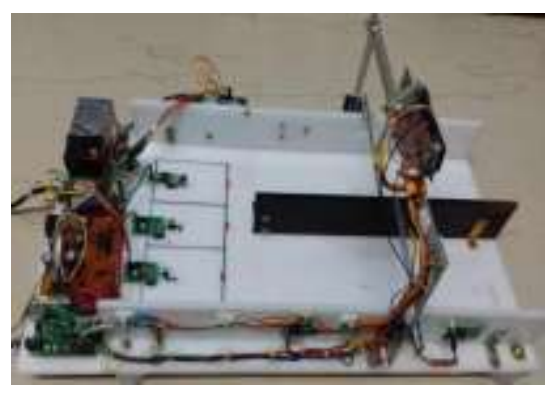

Figure 7: Top view of prototype model

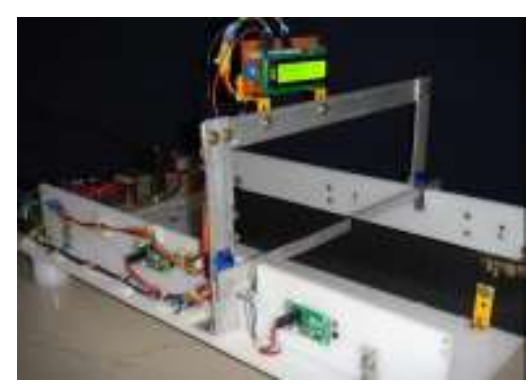

Figure 9. Entry IR sensor $1 \&$ 2, Entry servo motor

\section{Conclusion}

Hence the idea of automating the process of intelligent parking system will enhance better parking process. The mechanism works on a simple principle and there is not much complexity needed in the circuit and also works on low cost. The accuracy of this project may be improved by adding some features. The system was helpful over reduce the wasting time of searching parking lot and also improving the parking lot estimation. This paper outlines the basic idea of developing a system to handle parking related issues in bigger Malls/ shopping complex. For a bigger parking system, detailed engineering has to be done considering safety and other issues. By introducing Radio frequency identification tags on vehicles, automated billing system can be developed based on the duration of stay in the parking slot. 


\section{References}

1. H.G. Jung, D.S .Kim, J.Kim, Light-stripe-projection-based target position designation for intelligent parking-assist system, IEEE Transactions on Intelligent Transportation Systems, 2010 Dec; 11(4):94253.

2. Suhr, J. K., \& Jung, H. G. (2014), Sensor fusion-based vacant parking slot detection and tracking, IEEE Transactions on Intelligent Transportation Systems, 15(1), 21-36.

3. Jung HG, Cho YH, Yoon PJ, Kim J, Scanning laser radar-based target position designation for parking aid system, IEEE Transactions on Intelligent Transportation Systems, 2008 Sep;9(3) pp:406-24.

4. Wada M, Yoon KS, Hashimoto $\mathrm{H}$, Development of advanced parking assistance system, IEEE Transactions on Industrial Electronics, 2003 Feb;50(1) PP:4-17.

5. Kokolaki E, Karaliopoulos M, Stavrakakis I. Leveraging information in parking assistance systems, IEEE Transactions on Vehicular Technology, 2013 Nov;62(9)pp:4309-17.

6. Barone RE, Giuffrè T, Siniscalchi SM, Morgano MA, Tesoriere G, Architecture for parking management in smart cities, IET Intelligent Transport Systems, 2013 Dec 6;8(5)pp:445-52.

7. Sun DJ, Ni XY, Zhang LH, A discriminated release strategy for parking variable message sign display problem using agent-based simulation, IEEE Transactions on Intelligent Transportation Systems, 2016 Jan;17(1)pp:38-47. 